\title{
Ipilimumab induced digital vasculitis
}

\author{
Amrita Padda ${ }^{1 *}$ (D) Elena Schiopu', Justin Sovich², Vincent $\mathrm{Ma}^{2}$, Ajjai $^{1}$ Alva $^{3}$ and Leslie Fecher ${ }^{3}$
}

\begin{abstract}
Background: Immune check point inhibitors (ICls) have emerged as a new therapeutic paradigm for a variety of malignancies including metastatic melanoma. As the use of ICls expand, immune-mediated adverse events are becoming a common occurrence.

Case presentation: We describe the first reported patient with small vessel vasculitis, manifested by digital ischemia, following treatment with high dose Ipilimumab for resected stage IIIB/C melanoma. This patient received high dose steroids, five-day intravenous (IV) Epoprostenol protocol, botulinum toxin injections, and Rituximab 375 mg/m² weekly for four cycles. With this treatment regimen, the digital ischemia did not progress proximally, but she did require multiple distal digit amputations about six months after the onset of her symptoms.

Conclusions: Prompt identification and management of immune related adverse events (IRAEs) are critical to optimal patient management. This patient's vasculitis did not reverse, but was likely halted and stabilized with multiple immunosuppressive medications.
\end{abstract}

Keywords: Ipilimumab, Immune related adverse events (IRAEs), Vasculitis

\section{Background}

Ipilimumab $\left(\right.$ Yervoy $\left.^{\circ}\right)$ is approved by the Food and Drug Administration (FDA) for the treatment of resected stage III melanoma and advanced unresectable melanoma. It is a fully human monoclonal antagonistic antibody which targets cytotoxic T lymphocyte antigen 4 (CTLA-4) on T cells and blocks the CTLA-4 interaction with its ligand CD80. CTLA-4 is an immune check point molecule which downregulates pathways of $\mathrm{T}$ cell activation. Therefore, when CTLA-4 is blocked with Ipilimumab, the T lymphocyte inhibitory pathway is hindered, and the immune response is enhanced, allowing $\mathrm{T}$ lymphocytes to destroy cancer cells [1]. Melanoma incidence continues to rise and metastatic melanoma results in approximately 53,000 deaths per year worldwide as estimated by the World Health Organization [2]. Ipilimumab was the first therapeutic agent to demonstrate an overall survival benefit in the treatment of advanced, unresectable melanoma [3]. It is currently approved by the FDA at a dose of $3 \mathrm{mg} / \mathrm{kg}$ in the metastatic setting. More recently, Ipilimumab $10 \mathrm{mg} /$ $\mathrm{kg}$ demonstrated an improved median relapse free survival

\footnotetext{
* Correspondence: rithydpu@gmail.com; amritapa@med.umich.edu 1Division of Rheumatology, Department of Internal Medicine, University of Michigan, Floor 3, Reception A, 1500 E Medical Center Drive, SPC 5342, Ann Arbor, MI 48109, USA

Full list of author information is available at the end of the article
}

of 26.1 months compared to 17.1 months for placebo in resected stage III cutaneous melanoma in the European Organization for Research and Treatment of Cancer (EORTC)18,071; this study led to its approval by the FDA for this indication [4]. An update for this study was recently published and reported a five year relapse free survival of $40.8 \%$ in the Ipilimumab group compared to $30.3 \%$ in the placebo group, with a median follow up of 5.3 years [5]. Five-year overall survival in the Ipilimumab group was $65.4 \%$ versus $54.4 \%$ in the placebo group. No vascular toxicities of any grade were reported. Please see Table 1 for adverse events.

Preliminary safety data from an unplanned interim analysis for Ipilimumab-treated subjects was recently presented from the 1609 trial sponsored by the Eastern Cooperative Oncology Group at the American Society for Clinical Oncology [6]. This phase III study in subjects with resected stage III and IV melanoma randomized 1673 patients to high dose interferon (HDI), Ipilimumab $3 \mathrm{mg} /$ $\mathrm{kg}$, or Ipilimumab $10 \mathrm{mg} / \mathrm{kg}$, with co-primary endpoints of relapse free survival and overall survival. They reported safety data for 1019 subjects treated at either dose of Ipilimumab, as well as relapse free survival data for 773 concurrently randomized subjects with a median follow up of 3.1 years. There were two deaths $(0.4 \%)$ in the lower dose Ipilimumab arm due to colitis and eight $(1.6 \%)$ in the 
Table 1 Grade 3/4/5 toxicities from the E1609 trial and EORTC trial. These studies are in the setting of resected patients (adjuvant)

\begin{tabular}{|c|c|c|c|c|}
\hline \multirow[b]{2}{*}{ Treatment type } & \multicolumn{2}{|c|}{$\begin{array}{l}\text { E1609 } \text { trial }^{a}(\text { Safety Data } n=1019) \\
(\text { Total Enrollment }=1673)\end{array}$} & \multicolumn{2}{|c|}{ EORTC 18071 trial $(n=945)$} \\
\hline & Ipi 3 mg/kg & Ipi 10 mg/kg & Ipi 10 mg/kg & Placebo \\
\hline Number of patients & 516 & 503 & 471 & 474 \\
\hline Adverse event of any grade & $98.4 \%$ & $100 \%$ & (465 99\%) & $432(91 \%)$ \\
\hline Treatment-related AE (any grade) & $96 \%$ & $98.8 \%$ & & \\
\hline Grade 3 adverse events ${ }^{b}$ & \multirow{2}{*}{$37 \%$} & \multirow{2}{*}{$57 \%$} & & \\
\hline Grade 4 adverse events ${ }^{\mathrm{b}}$ & & & & \\
\hline Immune related adverse events (grade 3/4) & $18.8 \%$ & $34 \%$ & $196(41.6 \%)$ & $13(2.7 \%)$ \\
\hline Gastrointestinal adverse event ${ }^{b}$ & $12.0 \%$ & $18.5 \%$ & $76(16 \%)$ & $4(<1 \%)$ \\
\hline Hepatic adverse events ${ }^{b}$ & $3.1 \%$ & $7.8 \%$ & $51(11 \%)$ & $1(<1 \%)$ \\
\hline Endocrine adverse events ${ }^{\mathrm{b}}$ & $6.6 \%$ & $12.4 \%$ & $37(8 \%)$ & $1(<1 \%)$ \\
\hline Neurologic adverse events ${ }^{b}$ & $2.0 \%$ & $1.6 \%$ & $9(1.9 \%)$ & $0(0 \%)$ \\
\hline $\begin{array}{l}\text { Treatment related Adverse event leading to } \\
\text { discontinuation of treatment }\end{array}$ & $35 \%$ & $54 \%$ & 240 (51\%) & $22(4.6 \%)$ \\
\hline Death due to treatment related adverse events & $2(0.4 \%)$ & $8(1.6 \%)$ & $5(1.1 \%)$ & 0 \\
\hline
\end{tabular}

${ }^{a}$ Abstract available only for the E1609 trial

${ }^{\mathrm{b}}$ Grade $3 / 4$ adverse events

higher dose Ipilimumab arm: five subjects with colitis, one pneumonitis, one thromboembolic event with hypophysitis, and one cardiac event. This unplanned exploratory analysis showed no difference in relapse free survival between the low dose and high dose Ipilimumab, however additional follow up is needed. Of note, the Ipilimumab $10 \mathrm{mg} / \mathrm{kg}$ arm accrual was suspended for approximately 2 months due to toxicity (Table 1 ). These adjuvant toxicity rates are at least equivalent and possibly greater than those reported in the advanced, unresectable melanoma setting with grade $3 / 4$ rates reported at $27-58 \%$ for $10 \mathrm{mg} / \mathrm{kg}$ [7-10].

\section{Case presentation}

We report a case of a 52-year-old Caucasian woman who was diagnosed with stage IIIB/C melanoma from a regressed primary of the abdomen when she presented with a bulky left groin mass. Imaging demonstrated a $6.5 \mathrm{~cm}$ lobulated mass in the left groin involving soft tissue and probable lymph nodes. Excisional biopsy revealed two lymph nodes with metastatic melanoma; tumor cells were positive for Melan-A and SOX-10, and negative for CD45, Cytokeratin AE1/AE3. The patient reported a long standing black skin lesion at the lower abdomen that had previously grown and bled, but more recently had started to fade. This lesion was biopsied and demonstrated superficial dermal fibrosis with tumoral melanosis and pigment laden macrophages (melanophages), consistent with a regressed melanocytic lesion. She had no distant metastases on staging and underwent a wide local excision of the regressed primary site and radical resection of the inguinal mass with complete lymph node dissection, followed by reconstruction of the left groin with a sartorius flap. Pathology of the $8.9 \mathrm{~cm}$ subcutaneous mass revealed a spindle cell (sarcomatoid) melanoma involving lymph node tissue, possible aggregate of matted lymph nodes with extranodal extension, and multiple additional lymph nodes without melanoma. She had no known history of autoimmune conditions or prior Raynaud's phenomenon.

The first high dose Ipilimumab of $10 \mathrm{mg} / \mathrm{kg}$ was administered at an outside hospital followed by limited loose bowel movements and mouth soreness. One week after the second Ipilimumab infusion (at 3-week intervals), she developed myalgias, arthralgias, rash, vision changes, jaw pain, and discoloration of several upper and lower limb digits. Initially, the digital symptoms included intermittent red and blue color changes, worse in the cold, and better with warmth. Two weeks after the second infusion, her oncologist initiated Amlodipine $10 \mathrm{mg}$ daily, Aspirin $81 \mathrm{mg}$ daily, and Prednisone $10 \mathrm{mg}$ daily for suspected immunemediated Raynaud's phenomenon. Serologic workup at the initial presentation included a negative antinuclear antibody, rheumatoid factor, anti-cyclic citrullinated peptide antibody, cytoplasmic and perinuclear anti-neutrophil cytoplasmic antibodies, cryoglobulins, and hepatitis C antibody and ribonucleic acid (RNA); serum and urine protein electrophoresis were within normal limits. She reported immediate improvement of the myalgias, rash, arthralgias and jaw pain, but she had progression of digital pain and discoloration, therefore her oncologist administered $500 \mathrm{mg}$ of IV Methylprednisolone and increased her daily Prednisone to $60 \mathrm{mg}$ for a presumed Ipilimumab immune related adverse event (IRAE). Her digits did not improve, and she received a second $500 \mathrm{mg}$ dose of IV 
Methylprednisolone three days later. The lower extremity digital pain resolved, but the upper extremity digital pain became so severe that she required high doses of morphine.

She was admitted for inpatient management two days after her outpatient steroid infusions. Physical exam revealed acrocyanosis of all upper extremity digits (Fig. 1a \& 1b). She was initiated on $2 \mathrm{mg} / \mathrm{kg}$ of IV Methylprednisolone and calcium channel blockade. Lower extremity digits were less affected and demonstrated the greatest improvement. The upper extremity digital pain and acrocyanosis persisted. She was treated with one dose of $1000 \mathrm{mg}$ of IV Methylprednisolone with limited benefit, and thus continued daily $2 \mathrm{mg} / \mathrm{kg}$ dosing. Arterial duplex dopplers of upper and lower extremities were normal. Transthoracic echocardiogram was negative for thrombus or mass. Conventional angiogram was performed on the left upper extremity; it revealed severely diminished blood flow in the digital arteries beyond the proximal interphalangeal joints, and the Verapamil challenge did not increase blood flow (Fig. 2). Findings on the conventional angiogram were consistent with small vessel occlusive disease. Extensive autoantibody panels (inclusive of myeloperoxidase, proteinase 3, antinuclear, anti-centromere, anti-topoisomerase I, Beta-2-glycoprotein 1, anti-cardiolipin, anti-neutrophil cytoplasmic) and viral panels (human immunodeficiency virus, hepatitis panels) were negative. A full coagulopathy work-up was unrevealing. Given concern for ongoing immune-

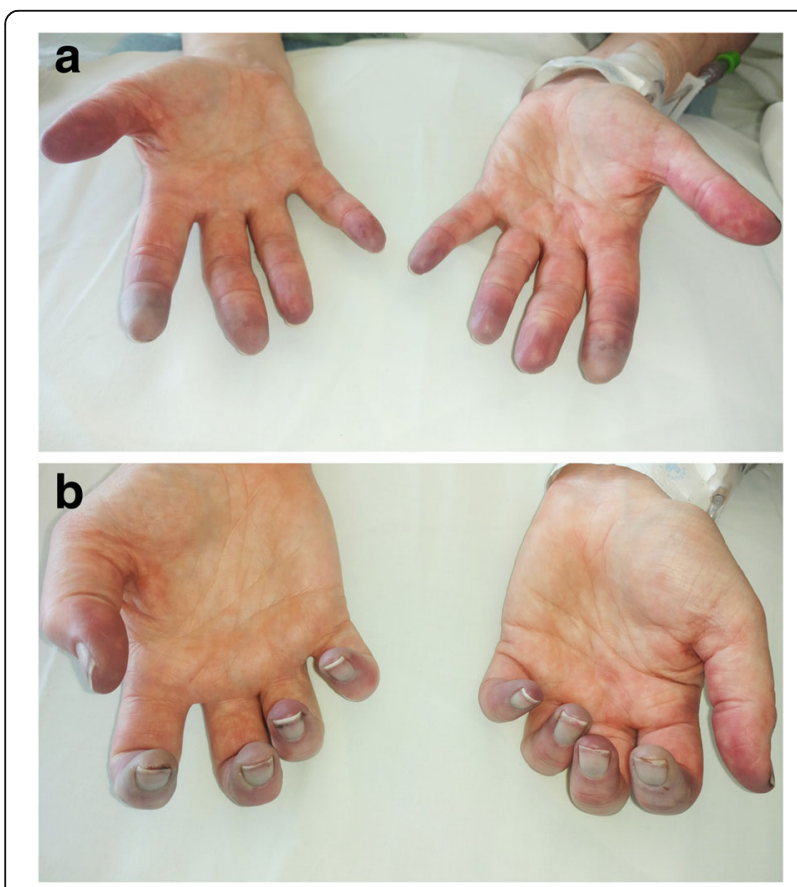

Fig. 1 (Panel $\mathbf{a}$ and $\mathbf{b}$ ). This picture was taken four weeks after her second Ipilimumab infusion (week 26 on timeline). Physical exam reveals acrocyanosis of all digits with small ulcers of the right second and fourth fingertips

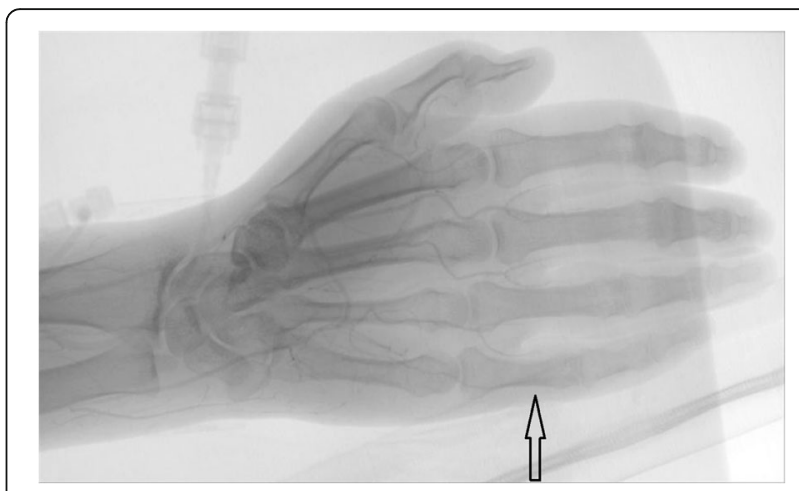

Fig. 2 Conventional angiogram of the left arm was performed five weeks after her second Ipilimumab infusion (end of week 26 on timeline). There is severely diminished flow in the digital arteries of the left hand beyond the level of all proximal interphalangeal joints (black arrow), consistent with small vessel occlusive disease

mediated vasculitis, the patient also received a 5-day course, 6-h per day, of Epoprostenol at a rate of $3 \mathrm{ng} / \mathrm{kg} / \mathrm{min}$. Fifty units of botulinum toxin $\mathrm{A}$ in $10 \mathrm{~mL}$ of normal saline was injected into each hand via palmar approach focusing on the proximal aspect of each digital artery. After six days of IV steroids, she was transitioned to oral Prednisone $100 \mathrm{mg}$ $(1 \mathrm{mg} / \mathrm{kg}$ ) daily. She received four cycles of Rituximab at $375 \mathrm{mg} / \mathrm{m}^{2}$, at approximately one-week intervals. Her Prednisone was tapered to $10 \mathrm{mg}$ daily over the course of seven weeks. She then developed an IRAE of pneumonitis, which quickly improved when the Prednisone was increased to $50 \mathrm{mg}$ daily. During steroid treatment, she was also on Alendronate and calcium for osteoporosis prophylaxis, Bactrim for pneumocystis pneumonia prophylaxis, and Omeprazole for gastrointestinal protection.

After the four cycles of Rituximab, we believe that the vasculitic process and additional damage were halted, as she did not develop further proximal digital ischemia or other systemic symptoms. The exam appeared worse with dry gangrene of the fingertips, consistent with the natural evolution of skin changes with distal digital ischemia, as shown in Fig. 3a \& b (Taken 5 weeks after Fig. 1), likely reflecting consequences of the initial injury. She did ultimately require distal digit amputations. See Table 2 for the chronological outline of the case presentation.

\section{Discussion and conclusions}

Immune related adverse events (IRAEs) are common with checkpoint inhibitors, and can range from mild to severe and life threatening $[11,12]$. While there are common patterns in presentation, it is not possible to predict specific toxicities at this time. Skin, gastrointestinal tract, liver and endocrine glands are commonly affected. In general, IRAEs are treated with steroids which can require high doses with slow tapers at least over one 

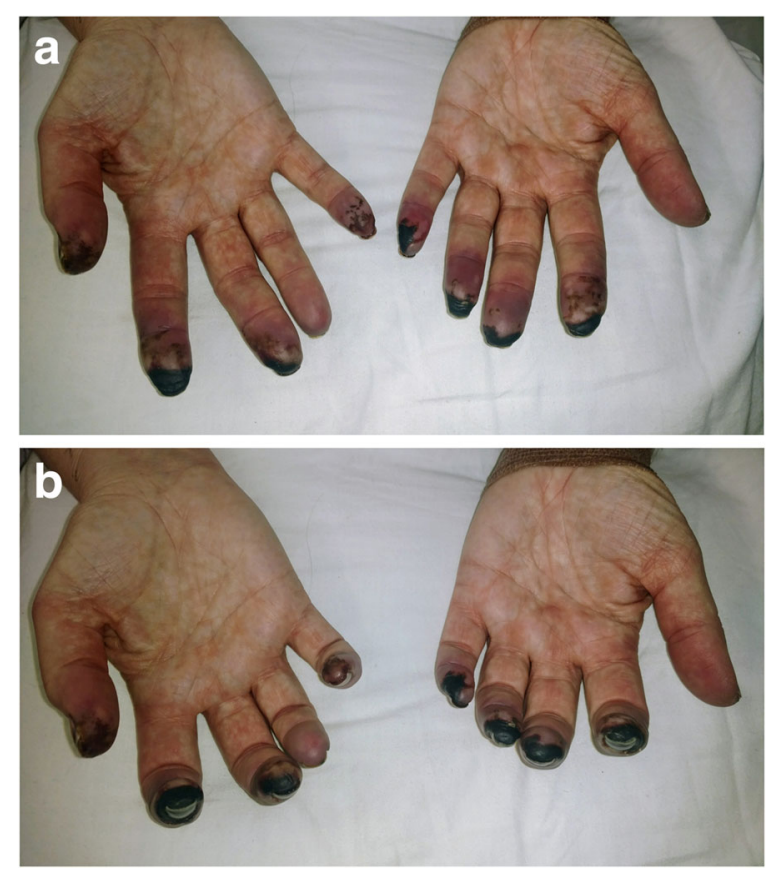

Fig. 3 (Panel $\mathbf{a}$ and $\mathbf{b}$ ) This picture was taken nine weeks after her second Ipilimumab infusion (week 31 on timeline). The patient is status-post high dose steroids and four cycles of Rituximab. The exam appeared worse with dry gangrene of the fingertips, secondary to the natural evolution of skin changes with distal digital ischemia. We believe that the vasculitic process was halted, as she did not develop further proximal digital ischemia

month. Some IRAEs can be refractory and require additional immunosuppressive or immune-modulating agents including Infliximab or Mycophenolate [11-13]. Immune-related toxicities have been reported involving nearly all organ systems, including rare cardiac and neurologic toxicities [14]. Inflammatory arthritis mimicking rheumatoid arthritis and sicca syndrome have frequently been described in Ipilimumab use [15]. However, IRAEs involving blood vessels, such as vasculitides are quite rare and reported at a less than $1 \%$ incidence [16]. The most common vascular IRAE reported to date is giant cell arteritis/temporal arteritis [17]. A recent review addresses the molecular mechanism of how immune checkpoint inhibitors increase anti-vascular immunity risk, specifically in causing medium and large cell vasculitis, like giant cell arteritis [18]. In a search across PubMed, there have only been three case reports of vasculitis in Ipilimumab use. One case was isolated lymphocytic uterine vasculitis [19]. The other two cases were polymyalgia rheumatica with giant cell arteritis, both patients highly responsive to prednisone [20].

To our knowledge, this is the first reported case of an Ipilimumab IRAE causing small vessel vasculitis manifesting as digital ischemia. This case illustrates the current debate in melanoma oncology regarding the risk-benefit
Table 2 Summarized timeline of case presentation

Week $0 \quad$ Presented with left groin mass. Excisional lymph node biopsy was consistent with metastatic melanoma.

Week 4 Radical resection of melanoma with wide local excision of regressed primary and complete lymph node dissection.

Week $19 \quad$ First cycle of Ipilimumab 10 mg/kg. Side effects included mild diarrhea and mouth soreness.

Week 22 Second cycle of Ipilimumab $10 \mathrm{mg} / \mathrm{kg}$.

Week 23 Symptoms of myalgias, arthralgias, rash, vision changes, jaw pain, and discoloration of several upper and lower limb digits.

Week 24 Amlodipine $10 \mathrm{mg}$ daily, Aspirin $81 \mathrm{mg}$ daily, and Prednisone $10 \mathrm{mg}$ daily initiated for suspected Raynaud's phenomenon. Digital pain and discoloration progressed.

Week 25 She received Methylprednisolone $500 \mathrm{mg}$ IV followed by oral Prednisone $60 \mathrm{mg}$ daily. Additional dose of $500 \mathrm{mg}$ Methylprednisolone IV given later in the week. Lower extremity digital pain resolved, upper extremity digital pain progressed.

Week 26 Admitted. Initiated on Methylprednisolone 2 mg/kg/day IV, calcium channel blockade, and nitropaste. Administered an additional Methylprednisolone $1000 \mathrm{mg}$ dose. Epoprostenol initiated for a 5-day course. Botulinum toxin A was injected into each hand. Refer to Fig. 1 for the physical exam and Fig. 2 for the conventional angiogram.

Week 27 Transitioned to oral Prednisone $100 \mathrm{mg}(1 \mathrm{mg} / \mathrm{kg})$ daily and Sildenafil $20 \mathrm{mg}$ BID.

Week 27-30 Initiated on weekly Rituximab 375 mg/m2. Refer to Fig. 3 for the physical exam at week 31.

Week 32 Prednisone was tapered down to $10 \mathrm{mg}$ daily.

Week 34 Developed IRAE of pneumonitis, prednisone was increased to $50 \mathrm{mg}$ daily and symptoms improved.

Week 48-52 Surgical amputation of multiple distal digits.

Week 49 Prednisone completely weaned off.

profile of adjuvant high dose Ipilimumab. Multiple studies of programmed death receptor-1 inhibitors in the adjuvant setting are completed with preliminary data or ongoing with pending data and further data from ECOG 1609 trial is forthcoming. This case highlights the importance of close monitoring of patients on immune checkpoint inhibitor therapy and prompt diagnosis and management of immune related adverse events.

\section{Abbreviations}

CTLA-4: Cytotoxic T-lymphocyte antigen 4; ICI: Immune checkpoint inhibitor; IRAE: Immune related adverse event; IV: Intravenous

\section{Acknowledgements \\ N/A}

Funding

N/A

Availability of data and materials

N/A 


\section{Authors' contributions}

$A P, L F, J S, V M, A A$ all provided care to this patient. AP, LF, ES all made significant contributions to drafting the manuscript. JS, AA, and VM revised and edited the manuscript multiple times. Each author has given final approval of the version to be published. All authors have agreed to be accountable for all aspects of the work in ensuring that questions related to the accuracy or integrity of any part of the work are appropriately investigated and resolved. All authors read and approved the final manuscript.

\section{Ethics approval and consent to participate}

No formal ethics approval was needed since we were only reporting an observational case report. Consent was obtained from the patient.

\section{Consent for publication}

Consent was obtained from the patient. She signed a biomed generic consent for this journal.

\section{Competing interests}

The authors declare that they have no competing interests.

\section{Publisher's Note}

Springer Nature remains neutral with regard to jurisdictional claims in published maps and institutional affiliations.

\section{Author details}

'Division of Rheumatology, Department of Internal Medicine, University of Michigan, Floor 3, Reception A, 1500 E Medical Center Drive, SPC 5342, Ann Arbor, MI 48109, USA. ${ }^{2}$ Department of Internal Medicine, University of Michigan, Ann Arbor, MI, USA. ${ }^{3}$ Division of Hematology Oncology,

Department of Internal Medicine, University of Michigan, Ann Arbor, MI, USA.

Received: 1 August 2017 Accepted: 23 January 2018

Published online: 12 February 2018

\section{References}

1. Melero I, Hervas-Stubbs S, Glennie M, Pardoll DM, Chen L. Immunostimulatory monoclonal antibodies for cancer therapy. Nat Rev Cancer. 2007;7:95-106

2. World Health Organization. Skin cancers. (Accessed 1 May 2017, at http:// www.who.int/uv/faq/skincancer/en/index1.html.)

3. Hodi FS, O'Day SJ, McDermott DF, et al. Improved survival with ipilimumab in patients with metastatic melanoma. N Engl J Med. 2010;363(8):711-23.

4. Eggermont AM, Chiarion-Sileni V, Grob JJ, et al. Adjuvant ipilimumab versus placebo after complete resection of high-risk stage III melanoma (EORTC 18071): a randomized, double-blind, phase 3 trial. Lancet Oncol. 2015:16(5):522-30.

5. Eggermont AM, Chiarion-Sileni V, Grob JJ, et al. Prolonged survival in stage III melanoma with Ipilimumab adjuvant therapy. N Engl J Med. 2016;375(19): 1845-55.

6. Tarhini AA, Lee SJ, Hodi FS, et al. A phase III randomized study of adjuvant ipilimumab (3 or $10 \mathrm{mg} / \mathrm{kg}$ ) versus high-dose interferon alfa-2b for resected high-risk melanoma (U.S. Intergroup E1609): Preliminary safety and efficacy of the ipilimumab arms. J Clin Oncol. 2017 (suppl; abstr 9500).

7. Wolchok JD, Neyns B, Linette G, et al. Ipilimumab monotherapy in patients with pretreated advanced melanoma: a randomized, double-blind, multicenter, phase 2, dose-ranging study. Lancet Oncol. 2010;11(2):155-64.

8. Ascierto PA, Del Vecchio $M$, Robert $C$, et al. Ipilimumab 10 mg/kg versus ipilimumab $3 \mathrm{mg} / \mathrm{kg}$ in patients with unresectable or metastatic melanoma: a randomized, double-blind, multicenter, phase 3 trial. Lancet Oncol. 2017;18(5):611-22.

9. Hodi FS, Lee S, McDermott DF, et al. Ipilimumab plus sargramostim vs ipilimumab alone for treatment of metastatic melanoma: a randomized clinical trial. JAMA. 2014;312(17):1744-53.

10. Weber J, Thompson JA, Hamid O, et al. A randomized, double-blind, placebocontrolled, phase II study comparing the tolerability and efficacy of ipilimumab administered with or without prophylactic budesonide in patients with unresectable stage III or IV melanoma. Clin Cancer Res. 2009;15(17):5591-8.

11. Fecher LA, Agarwala SS, Hodi FS, Weber JS. Ipilimumab and its toxicities: a multidisciplinary approach. Oncologist. 2013;18(6):733-43.
12. Weber JS, Postow M, Lao CD, Schadendorf D. Management of Adverse Events Following Treatment with Anti-Programmed Death-1 agents. Oncologist. 2016;21(10):1230-40.

13. Weber J. Ipilimumab: controversies in its development, utility and autoimmune adverse events. Cancer Immunol Immunother. 2009;58(5):823-30.

14. Michot JM, Bigenwald C, Champiat S, et al. Immune-related adverse events with immune checkpoint blockade: a comprehensive review. Eur J Cancer. 2016;54:139-48.

15. Cappelli LC, Gutierrez AK, Baer AN, et al. Inflammatory arthritis and sicca syndrome induced by nivolumab and ipilimumab. Ann Rheum Disease. 2017;76(1):43-50.

16. Ipilimumab FDA label. https://www.accessdata.fda.gov/drugsatfda_docs/ label/2011/125377s0000lbl.pdf.

17. O'Day SJ, Maio M, Chiarion-Sileni V, et al. Efficacy and safety of ipilimumab monotherapy in patients with pretreated advanced melanoma: a multicenter single-arm phase II study. Ann Oncol. 2010;21(8):1712-7.

18. Watanabe R, Zhang H, Berry G, Goronzy JJ, Weyand CM. Immune checkpoint dysfunction in medium and large vessel Vasculitis. Am J Physiol Heart Circ Physiology. 2017;312(5):H1052-9.

19. Minor DR, Bunker SR, Doyle J. Lymphocytic Vasculitis of the Uterus in a Patient with Melanoma Receiving Ipilimumab. J Clin Oncol. 2013;31(20):e356.

20. Goldstein BL, Gedmintas L, Todd DJ. Drug associated Polymyalgia Rheumatica/Giant cell Arteritis occurring in two patients after treatment with Ipilimumab, an antagonist of CTLA-4. Arthritis \& Rheumatology. 2014;66(3):768-9.

\section{Submit your next manuscript to BioMed Central and we will help you at every step:}

- We accept pre-submission inquiries

- Our selector tool helps you to find the most relevant journal

- We provide round the clock customer support

- Convenient online submission

- Thorough peer review

- Inclusion in PubMed and all major indexing services

- Maximum visibility for your research

Submit your manuscript at www.biomedcentral.com/submit
) Biomed Central 